\title{
Dafang Wu: Clinical Nuclear Medicine Neuroimaging: An Instructional Casebook
}

\author{
Springer Nature Switzerland AG, 2020, ISBN 978-3-030-40892-3
}

\author{
Eugenio Di Giorgio ${ }^{1} \cdot$ Luigi Mansi $^{2}$
}

Published online: 20 May 2021

(C) The Author(s), under exclusive licence to Springer-Verlag GmbH Germany, part of Springer Nature 2021

Nowadays, neurological diseases are becoming a growing public health problem, also because the lengthening of the average life has increased the percentage and the total number of elderly subjects. Therefore, remaining mandatory and primary position of neuro-radiological techniques, i.e., computed tomography (CT) and magnetic resonance (MRI), the role of nuclear medicine is increased, mainly in the field of neurodegenerative diseases, and in particular in the patients suspicious for dementia.

The space available to radionuclide procedures has enlarged in recent years also due to the availability and/or to a wider diffusion of more and more performing hybrid scanners, i.e., PET/CT, PET/MRI, SPECT/CT, and of new radiotracers capable of studying perfusion, metabolism, receptor status, and other pathophysiological conditions.

In this scenario, an adequate technical and interpretative knowledge of radionuclide neuroimaging appears necessary at first for the neurologist and the other clinicians interested in neuro-psychiatric diseases, who needs to fully understand the potential and the indications of these procedures. Similarly, becoming familiar with these techniques is mandatory for all the professionals involved in nuclear medicine, who needs to apply at the best the radioisotopic methods to answer to the clinical queries giving relevance to their role in this specific clinical context.

This publication has been edited to reach these goals by Dafang $\mathrm{Wu}$, Professor in the Department of Diagnostic Radiology and Molecular Imaging at Oakland University William Beaumont School of Medicine.

Structured in 379 pages, enriched by 127 illustrations, the casebook consists of eight chapters, concerning the role of both

Luigi Mansi

Mansi.luigi@libero.it

1 Nuclear Medicine Department, Ospedale del Mare, Naples, Italy

2 Inter-University Research Center for Sustainability (CIRPS), Rome, Italy
SPECT/CT and PET/CT in the study of dementias and epilepsies, of dopamine transporter scan in Parkinson's disease and parkinsonisms, also paying attention to the role PET-FDG in neuro-oncology. The scintigraphic study of the cerebrospinal fluid, perfusion scans in the evaluation of the diagnosis of brain death, and other less diffuse applications are also reported. More precisely, the book is organized in the following chapters: (1) Positron Emission Tomography (PET) in Dementias, (2) Single Photon Emission Computed Tomography (SPECT) in Dementias, (3) F-18 Fluorodeoxyglucose Positron Emission Tomography (FDG PET) in Epilepsies, (4) Single Photon Emission Computed Tomography (SPECT) in Epilepsies, (5) FDG PET Imaging of Brain Tumors, (6) Dopamine Transporter Scan (DaTscan), (7) Cerebrospinal Fluid (CSF) Scintigraphy, and (8) Miscellaneous Clinical Nuclear Medicine Neuroimaging Studies.

Each chapter is comprehensive with case presentation based on pertinent clinical information, representative images with illustrations, key imaging findings, concise discussion/ follow-up data, and with a case summary as conclusion.

At the end of this book, two appendices are included, SelfAssessment Quiz and Imaging Protocols, which are provided for those who wish to test their own ability in the interpretation of nuclear medicine neuroimaging studies, in order to stimulate the criticality of the readers.

Although the volume does not include all the most recent techniques and procedures based on radiotracers, it can be helpful at first for residents and practitioners in nuclear medicine, finding a possible role also for clinicians involved in neurosciences. It can be a good start mainly for beginners, with main reference to young physicians in preparation for their in-service tests and board examinations.

Publisher's note Springer Nature remains neutral with regard to jurisdictional claims in published maps and institutional affiliations. 